\title{
Decomposing Complex Cooperative Ligand Binding into Simple Components: Connections between Microscopic and Macroscopic Models
}

\author{
Alexey Onufriev ${ }^{\dagger}$ and G. Matthias Ullmann*,* \\ Department of Computer Science, 660 McBryde Hall, Virginia Tech, Blacksburg, Virginia 24061, and \\ Structural Biology/Bioinformatics, University of Bayreuth, Universitätsstr. 30, BGI, 95447 Bayreuth, Germany
}

Received: January 5, 2004; In Final Form: March 30, 2004

\begin{abstract}
Cooperative ligand-binding curves may often appear deceptively featureless, yet the underlying microscopic models may be rather complex, and the connection between them is not intuitive. To address some of these issues, we have extended the framework of the decoupled sites representation (DSR), previously developed in the context of $\mathrm{pH}$ titration, to include cooperative ligand binding as well as multiple conformations and multiple ligands. The extended framework is based on general thermodynamic arguments and is applicable to both anti-cooperative and cooperative binding. It can be used to elucidate the connection between the experimentally observed binding curves and parameters of underlying microscopic models. It is demonstrated that any binding curve can be decomposed into simple standard components that permit a model-independent physical interpretation in terms of noninteracting (quasi) groups. A simple mathematical form of the DSR is proposed, that is well-suited for use in least-squares fitting of experimental binding curves; the fitting procedure produces an integer parameter indicative of the degrees of cooperativity possible in the system. A two-site example is worked out in detail. We demonstrate that the same macroscopic binding behavior observed experimentally can have qualitatively different origins at the level of the underlying microscopic mechanism. We also show that, in the absence of the microscopic model, it is not possible to draw a meaningful distinction between non-cooperative and anti-cooperative scenarios. We define a new measure of cooperativity and show that it is in many cases more adequate than the Hill coefficient when used to characterize complex binding curves. The extended DSR is applied to experimental data sets on oxygen binding to carp hemoglobin at different $\mathrm{pHs}$, where the framework is used to interpret the degree of cooperativity in the system and provides an indication as to whether specific microscopic models are applicable.
\end{abstract}

\section{Introduction}

Binding of ligands to macromolecules is one of the most important reactions in biology; well-known examples include oxygen binding to hemoglobin, calcium binding to calmodulin, and magnesium binding to RNA. Often binding of one ligand molecule can enhance or reduce the receptor's affinity to bind subsequent ligand molecules. Such effects are in general referred to as "cooperative", and can be found in many biological systems. Reduction of affinity upon multiple ligand binding can be attributed to the presence of an effective repulsion between the ligands and is called negative cooperativity or anticooperativity. Affinity enhancement due to ligand binding can be attributed to an effective attraction between the ligands and is called positive cooperativity or simply cooperativity. Positive cooperativity is important for the regulation of biochemical reactions, ${ }^{1,2}$ where it leads to steep titration curves and thus to a fast transition from the completely free to the completely bound state of the macromolecule as the ligand concentration is increased. Cooperative effects are also important for enzymes, where they can increase the enzymes' turnover rates $^{3}$ as in aspartate transcarbamylase, which was studied intensively.

* To whom correspondence may be addressed. Email: Matthias.Ullmann@uni-bayreuth.de. Fax: +49-921-55-3544.

$\dagger$ Virginia Tech.

$\doteqdot$ University of Bayreuth.
Because of its biological importance, cooperative binding has for a long time been attracting attention in the theoretical biochemistry community. Adair was probably one of the first to develop a quantitative model of cooperative ligand binding. ${ }^{4}$ It was based on general thermodynamic arguments and did not rely on any microscopic or structural details of the binding process. Much later, Monod, Wyman, and Changeux ${ }^{5}$ as well as Koshland, Némethy, and Filmer ${ }^{6}$ developed their treatment of cooperative binding based on an underlying structural model. Hammes and $\mathrm{Wu}^{3}$ pointed out that the Monod-WymanChangeux (MWC) as well as the Koshland models were special cases of a more general model of cooperative binding. Over the past few decades, a great number of studies have been carried out aimed at understanding the interaction between ligands ${ }^{7-20}$ using both structure-based and phenomenological models.

In a our previous work, ${ }^{21}$ we discussed the complexity of titration curves that involve more than one interacting ligand and showed that any titration curve, no matter how complex, can be decomposed into a linear combination of simple standard components. These components are sigmoidal titration curves corresponding to noninteracting quasisites; the latter are characterized by their own set of binding constants and are related to the original microscopic binding sites via a linear transformation. The relationship between binding constants of the quasisites, the original (physical) binding sites, and the standard macroscopic binding constants is discussed in detail in ref 22 . 
The new framework, the decoupled site representation or DSR, ${ }^{21}$ allows us to interpret any complicated titration behavior in a model-free, thermodynamically rigorous way. The DSR is thus analogous to the normal-mode representation of vibrations. Previously, we focused on $\mathrm{pH}$ titration as a typical example of anti-cooperative ligand binding. While the general arguments used in the derivation of the DSR apply verbatim to the cooperative case as well, and the same mathematical formalism holds, the presence of an effective attractive interaction between the ligands can cause some of the quasisite binding constants to acquire an imaginary part. One way out of this difficulty would be to use the concept of "ghost site"1 with the associated binding constants that are allowed to have an imaginary part. However, as we showed in our previous work ${ }^{21}$ on the anticooperative scenario, the DSR quasisite binding constants can be directly measured experimentally and are straightforwardly related to the energetic parameters of the system. To treat both cooperative and anti-cooperative binding within the same framework, we have reworked the mathematical formalism of the DSR to accommodate the cooperative case without the explicit use of nonreal numbers for all the binding constants involved. This strategy is consistent with our philosophical conviction that a physical observable should be a purely real number. We will use cooperative oxygen binding to carp hemoglobin as a test case and will show how an application of the DSR helps to interpret experimental data and select plausible microscopic models.

We begin by briefly outlining the theory of multiple ligand binding and the concept of macroscopic and microscopic binding constants. We then introduce the DSR and the idea of quasisites and their binding constants. We extend the DSR framework to the multiple conformer case and consider binding of more than one type of ligands. We introduce the notion of a quasigroup, which is a generalization of the quasisite concept introduced earlier. Each quasigroup is characterized by a set of purely real binding constants analogous to macroscopic binding constants. We define a measure of cooperativity for each quasigroup and show that it is superior to the Hill coefficient when used to interpret cooperative binding curves. Furthermore, we show that a simple equation based on purely real numbers can be used to fit experimental data to the DSR model and that an integer parameter obtained automatically as a result of the least-squares fit can provide valuable information about types of cooperative behavior possible in the system. A two-site example is worked out in detail; we use this case, which permits an analytical solution, to demonstrate that very different underlying microscopic models may lead to the same phenomenological ligandbinding behavior at the macroscopic level. The DSR helps to unambiguously classify similar types of binding and distinguish between qualitatively different scenarios. We apply the extended DSR to available experimental data sets on oxygen binding to carp hemoglobin at different $\mathrm{pH}$, show how the framework can help to interpret the various degrees of cooperativity seen in the system, and provide an indication whether certain microscopic models (such as the two-state MWC) are applicable. In the end of the paper, we summarize our main conclusions. Details of some of the derivations, too lengthy for the main text, are presented in the Supporting Information.

\section{Theory}

Basic Concepts in Multiple Ligand Binding. In this section, we summarize the basic concepts in the theory of multiple ligand binding and connect two different approaches which often complement each other in theoretical studies. The first approach describes titration curves in terms of equilibrium constants; the second one uses the energies of micro- and macrostates. Both approaches are fully equivalent, but the notational framework of the latter is more compact for molecules that can bind many ligands.

The binding of a ligand $\mathrm{L}$ to a receptor $\mathrm{R}$ is described by the following basic equations

$$
\mathrm{R}+\mathrm{L} \stackrel{K}{\rightleftharpoons} \mathrm{RL} \quad \mathrm{K}=\frac{[\mathrm{RL}]}{[\mathrm{R}][\mathrm{L}]}
$$

The square brackets denote the concentration (activity) of the species. The standard energy $\Delta G^{\circ}$ of ligand binding is given by

$$
G^{\circ}=-\beta^{-1} \ln K
$$

with $\beta=\left(k_{\mathrm{B}} T\right)^{-1}$, where $k_{\mathrm{B}}$ is the Boltzmann constant and $T$ is the absolute temperature. The probability $\langle x\rangle$ of finding a ligand associated with its receptor at a given chemical potential $\mu_{\mathrm{L}}$ of the ligand is given by

$$
\langle x\rangle=\frac{\mathrm{e}^{-\beta\left(G^{\circ}-\mu_{\mathrm{L}}\right)}}{1+\mathrm{e}^{-\beta\left(G^{\circ}-\mu_{\mathrm{L}}\right)}}=\frac{K \Lambda}{1+K \Lambda}
$$

where $\Lambda=[\mathrm{L}]=\mathrm{e}^{\beta \mu_{\mathrm{L}}}$ is the ligand activity. This equation describes a sigmoidal titration curve with a unit slope at its only inflection point. In the following, we call such titration curves standard sigmoidal titration curves.

The ligand binding to a molecule that can bind $N$ ligands of the same type is given by

$$
\mathrm{R}+N \mathrm{~L} \stackrel{\bar{K}_{1}}{\rightleftharpoons} \mathrm{RL}+(N-1) \mathrm{L} \stackrel{\bar{K}_{2}}{\rightleftharpoons} \ldots \stackrel{\bar{K}_{N}}{\rightleftharpoons} \mathrm{RL}_{N}
$$

The species $\mathrm{RL}_{i}$ is the macrostate of the receptor with $i$ ligands bound. The total binding curve $\langle X\rangle$ is in general described by eq 5

$$
\langle X\rangle=\frac{\bar{K}_{1} \Lambda+2 \bar{K}_{1} \bar{K}_{2} \Lambda^{2}+\ldots+N \prod_{i=1}^{N} \bar{K}_{i} \Lambda^{N}}{1+\bar{K}_{1} \Lambda+\bar{K}_{1} \bar{K}_{2} \Lambda^{2}+\ldots+\prod_{i=1}^{N} \bar{K}_{i} \Lambda^{N}}
$$

The binding constants $\bar{K}_{i}$ are macroscopic binding constants for binding the $i$ th ligand to the molecule, i.e., the molecule is only considered as a whole, and individual binding sites are not considered explicitly. ${ }^{1,22}$ In contrast to macrostates, for which it is not specified to which sites the ligands bind, it is also possible to define microstates, for which this specification is required. For each macrostate with $i$ ligands bound, $\left({ }_{i}^{N}\right)=(N ! /$ $(N-i) ! i$ !) different microstates can be distinguished.

Rewriting eq 5 in terms of microscopic constants requires replacement of the macroscopic constants $\bar{K}_{i}$ in eq 5 by equivalent expressions based on microscopic constants..$^{9,23}$ Each microstate can be characterized by a state vector $\overrightarrow{\mathrm{x}}^{n}$, where each component $x_{i}^{n}$ is either 0 or 1 depending on whether the site $i$ is occupied or empty. For instance, a state of a three-site system in which the first and the third sites are occupied and the second one is empty is given by (101). The number of ligands bound to state $\mathbf{n}$ is given by $p_{\mathbf{n}}=\sum_{i=1}^{N} x_{i}^{n}$. The equilibrium between the microstate $\mathbf{u}$ (reactant state) and $\mathbf{v}$ (product state) is given by the microscopic equilibrium constant $k_{\mathbf{u}}^{\mathbf{v}}$. Writing eq 5 in terms of microscopic equilibrium constants would, however, 
result in rather complicated expressions even for systems with relatively few binding sites. ${ }^{9,23}$ We therefore switch to a different but equivalent representation, in which instead of using the equilibrium constants that lead to a certain microstate $\boldsymbol{n}$ from the reference state, we use the standard free energy $G_{\mathbf{n}}^{\circ}$ of this microstate relative to the reference state. The standard free energy $G_{\mathbf{n}}^{\circ}$ relates to the microscopic constants as given in eq 6

$$
G_{\mathbf{n}}^{\circ}=-\beta^{1} \ln \prod k_{\mathbf{u}}^{\mathbf{v}}
$$

The product in eq 6 ranges over the equilibria that connect the reference state with that $\mathbf{n}$. For example, for state (101), the product in eq 6 would be $k_{000}^{100} k_{100}^{101}$ or equivalently $k_{000}^{001} k_{001}^{101}$. The total binding curve in terms of the energies of the microstates is then given by eq 7

$$
\langle X\rangle=\frac{\sum_{j=1}^{2^{N}} p_{j} \mathrm{e}^{-\beta G^{\circ}} \Lambda^{p_{\mathrm{j}}}}{\sum_{j=1}^{2^{N}} \mathrm{e}^{-\beta G^{\circ}{ }_{j}} \Lambda^{p_{j}}}
$$

The denominator in eqs 5 and 7 corresponds to the grand canonical partition function $Z$ of the system.

The Decoupled Sites Representation for Multiple Conformers and More Than One Ligand. In the presence of an effective ligand-ligand interaction, titration curves of individual ligand-binding sites in a molecule can be considerably more complex than those of independent sites described by a standard sigmoidal curve. In our previous paper, ${ }^{21}$ we introduced a novel framework, the decoupled site representation (DSR), that decomposes any complicated titration behavior into simple standard (sigmoidal) components. The approach maps the set of $N$ interacting sites in the molecule onto a set of $N$ independent, noninteracting quasisites, each characterized by a new binding constant. From general thermodynamic arguments, we showed that the titration curve of an individual site in the molecule is a linear combination of standard sigmoidal titration curves corresponding to the quasisites. The total binding curve is the sum of these standard sigmoidal titration curves. The DSR allows to interpret any complicated titration behavior in a modelfree way.

In this section, we generalize the DSR to include multiple receptor conformations and allow for binding of more than one type of ligand. A more detailed derivation of the DSR for the case of a single conformation and one type of ligand can be found in ref 21.

Consider a molecule that can adopt $T$ conformations and has $N$ binding sites for ligand $\mathrm{L}$ and $R$ sites for another type of ligand S. Also different hydration levels of the receptor can be interpreted as alternative conformations of the receptor. The receptor is in equilibrium with the ligands in the surrounding solution. Only one single ligand can bind to one site. A ligand of type $\mathrm{L}$ cannot bind to a site for a ligand of type $\mathrm{S}$ and vice versa. The binding state of the molecule is specified by a $N+$ $R$ dimensional vector $\overrightarrow{\mathbf{x}}^{\mathbf{n}, \mathbf{r}, \mathbf{t}}$ where each element $x_{i}^{\mathbf{n}, \mathbf{r}, \mathbf{t}}$ is 1 or 0 depending on whether site $i$ is occupied or empty in the given conformation $\mathbf{t}$. The superscript $\mathbf{n , r}, \mathbf{t}$ denotes the microstate (ligand-binding and conformational states). We assume that sites from 1 to $N$ bind ligands of type $\mathrm{L}$ and sites from $N+1$ to $N$ $+R$ bind ligands of type $\mathrm{S}$. The total number of possible microstates of the molecule is $2^{N} \times 2^{R} \times T$. Each microstate is characterized by its free energy $G^{\mathbf{n}, \mathbf{r}, \mathbf{t}}$ and the number of bound ligands of each type, $p_{\mathbf{n}}=\sum_{i=1}^{N} x_{i}^{\mathbf{n}, \mathbf{r}, \mathbf{t}}$ and $q_{\mathbf{r}}=\sum_{j=N+1}^{R+N} x_{j}^{\mathbf{n}, \mathbf{r}, \mathbf{t}}$. Here, $G^{\mathbf{n}, \mathbf{r}, \mathbf{t}}$ is the standard Gibbs free energy of the microstate when all ligand activities equal 1 . In what follows, we take the reference state with free energy of zero to be the completely empty state of the lowest-energy conformer. The equilibrium properties of the system can be calculated from its grand canonical partition function, which can be written ${ }^{8,10}$ as a power series (binding polynomial) in the ligand activities $\Lambda_{\mathrm{L}}=\mathrm{e}^{\beta \mu_{\mathrm{L}}}$ and $\Lambda_{\mathrm{S}}=\mathrm{e}^{\beta \mu_{\mathrm{S}}}$

$$
Z=\sum_{\mathbf{t}=1}^{T} \sum_{\mathbf{r}=1}^{2^{R}} \sum_{\mathbf{n}=1}^{2^{N}} \mathrm{e}^{-\beta G^{n, r, t}} \Lambda_{\mathrm{L}}^{p_{n}} \Lambda_{\mathrm{S}}^{\mathrm{q}_{r}}
$$

The equilibrium occupation of the site $i$ averaged over all microstates is given by

$$
\left\langle x_{i}\right\rangle=\frac{1}{Z} \sum_{\mathbf{t}=1}^{T} \sum_{\mathbf{r}=1}^{2^{R}} \sum_{\mathbf{n}=1}^{2^{N}} x_{i}^{\mathbf{n}, \mathbf{r}, \mathbf{t}} \mathrm{e}^{-\beta G^{\mathbf{n}, \mathbf{r}, \mathbf{t}}} \Lambda_{\mathrm{L}}^{p_{n}} \Lambda_{\mathrm{S}}^{q_{r}}
$$

The calculation of the cooperativity between $\mathrm{L}$ and $\mathrm{S}$ requires an analysis of $Z$ as a function of both $\Lambda_{S}$ and $\Lambda_{L}$; thus instead of the problem of finding roots of a polynomial, it leads to the more complicated problem of finding the curves in the $\left(\Lambda_{S}, \Lambda_{L}\right)$ plane obtained from the intersection $Z\left(\Lambda_{\mathrm{S}}, \Lambda_{\mathrm{L}}\right)=0$. The latter equation is, in general, too complex and is unlikely to lead to a rigorous mathematical treatment that can be transparent enough to clarify the main points that follow. We therefore revert to the case of constant chemical potential of the ligand $S$ and consider it as a parameter, which is a typical experimental situation. This assumption reduces the partition function to the tractable form of the single-variable binding polynomial $Z\left(\Lambda_{\mathrm{L}}\right)$. In this case, eqs 8 and 9 are polynomials of $N$ th degree of only one variable $\Lambda=\Lambda_{\mathrm{L}}$, and so all the mathematical formalism developed in our earlier work ${ }^{21}$ applies. In particular, as was noted before, the binding polynomial eq 8 can always be factorized, ${ }^{1,8,9,24}$ and so we can rewrite eq 8 as

$$
Z=\prod_{i=1}^{N}\left[1+\Lambda_{\mathrm{L}} K_{i}^{\prime}\right]
$$

where $K_{i}^{\prime}$ is obtained as the negative inverse of the roots of the binding polynomial in eq 8 considering $\Lambda_{\mathrm{S}}$ constant. Applying the relationship ${ }^{25}$

$$
\sum_{i}\left\langle x_{\mathrm{i}}\right\rangle=\frac{\partial \ln Z}{\partial \ln \Lambda_{\mathrm{L}}}
$$

to eq 8 , one obtains for the total average occupancy of the molecule with $N$ ligands

$$
\langle X\rangle=\sum_{i=1}^{N}\left\langle x_{i}\right\rangle=\sum_{i=1}^{N} \frac{\Lambda_{\mathrm{L}} K_{i}^{\prime}}{1+\Lambda_{\mathrm{L}} K_{i}^{\prime}}
$$

Equation 12 as well as eq 10, from which it is derived, describes the binding to $N$ noninteracting or independent sites with some new binding constants $K_{i}^{\prime}$. We call these quasisites to distinguish them from the original, physical binding sites. The two sets become identical only when all ligand-binding sites are completely independent. Since it is a priori not known whether there is an effective interaction between the ligands, one cannot, from the total binding curve alone, assign $K_{i}^{\prime}$ to a particular physical site, although real-valued $K_{i}^{\prime} \mathrm{s}$ are experimentally accessible. $^{21}$ 
The original interacting binding sites are related to the quasisites by a linear transformation ${ }^{21}$

$$
\begin{aligned}
\left\langle x_{\mathrm{i}}\right\rangle & =\sum_{j}^{N} a_{i j}\left\langle y_{j}\right\rangle \\
\left\langle y_{j}\right\rangle & =\frac{K_{j}^{\prime} \Lambda_{\mathrm{L}}}{1+K_{j}^{\prime} \Lambda_{\mathrm{L}}}
\end{aligned}
$$

where $\left\langle y_{j}\right\rangle$ is the occupancy of quasisite $j$; the latter is described by a standard sigmoidal titration curve since, by construction, quasisites do not interact with each other. An explicit representation for the elements of the transformation matrix $\left\{a_{i j}\right\}$ can be found in our previous publication. ${ }^{21}$

The Decoupled Sites Representation for Cooperatively Interacting Sites. When the number of binding sites is $N>2$, there is no straightforward way ${ }^{8}$ to determine analytically the number of real roots of the binding polynomial eq 8. Our previous numerical analysis suggested ${ }^{21}$ that, in the single ligand/ single conformer case, repulsion between ligands on different sites is a sufficient condition for all of the roots to be purely real. Conversely, an effective attraction between the ligands may result in some of the roots being complex, which implies cooperativity between the sites. ${ }^{8}$ Cooperative binding means that the binding of a ligand increases the affinity for subsequent ligand binding. Such a scenario can be interpreted as an effective attraction between ligands. The opposite effect, i.e., the decrease of the ligand-binding affinity with the number of ligands already bound, is called anti-cooperative binding. We call binding non-cooperative if binding of a ligand has no effect on binding of the subsequent ligands. As we will show later, effective attraction, and therefore cooperativity, may result from conformational transitions after ligand binding, the presence of more then one type of ligand, or attractive site-site interactions.

In the case of cooperative binding, some of the quasisite binding constants become complex, i.e., they consist of a real and a nonzero imaginary part. For complex $K_{j}^{\prime}$, fitting of the total binding curve to eq 12 , although still mathematically possible with the use of complex $K_{j}^{\prime}$ and $a_{i j}$, is not practical. In addition, a physical interpretation of the complex binding constant is problematic.

To obtain a purely real expression which would not involve "complex binding constants", we follow Wyman's strategy and group the factors $\left[1+\Lambda K_{j}^{\prime}\right.$ in eq 8 to obtain a representation of $Z$ in terms of the product of lowest-degree polynomials with real, non-negative coefficients

$$
\begin{aligned}
Z= & \prod_{j=1}^{N_{1}}\left[1+K_{j}^{\prime} \Lambda\right] \times \prod_{j=1}^{N_{2}}\left[1+\bar{K}_{j}^{21} \Lambda+\bar{K}_{j}^{21} \bar{K}_{j}^{22} \Lambda^{2}\right] \times \ldots \times \\
& \prod_{j=1}^{N_{M}}\left[1+\bar{K}_{j}^{M 1} \Lambda+\bar{K}_{j}^{M 1} \bar{K}_{j}^{M 2} \Lambda^{2}+\ldots+\prod_{k=1}^{M} \bar{K}_{j}^{M k} \Lambda^{M}\right]
\end{aligned}
$$

where $N_{1}+2 N_{2}+\ldots+M N_{M}=N$. We call each of the above polynomials irreducible since, by construction, they cannot be further factored into lower-degree polynomials with real, nonnegative coefficients in front of $\Lambda^{k}$. Therefore, by construction, all $K_{j}^{\prime}$ and $\bar{K}_{j}^{m k}$ are real and positive and can be considered binding constants in the usual sense. The partition function in eq 15 describes a system that can be interpreted as a system of $N_{1}$ independent quasisites, $N_{2}$ pairs of cooperatively interacting quasisites, and so on up to $N_{M} m$ plets of cooperatively interacting quasisites $(1<m \leq M)$. We call the $m$ plets of cooperatively interacting quasisites quasigroups of order $m$ and the corresponding subpolynomial quasigroup polynomial. Quasigroups do not interact with each other since each corresponds to a factor in the partition function. The roots of the quasigroup polynomial are also roots of the whole binding polynomial. The constants $\bar{K}_{j}^{m k}$ are macroscopic binding constants of these quasigroups. It is not a priori possible to assign a given quasigroup to a real group of sites in the molecule. This situation is similar to the one of quasisites which cannot a priori be assigned to real sites in the molecule but, similar to normal modes, can include contributions from different sites.

By application of eq 11 to eq 15, one obtains for the total average occupancy of the molecule with $N$ ligands

$$
\begin{array}{r}
\langle X\rangle=\sum_{j}^{N_{1}} \frac{K_{j}^{\prime} \Lambda}{1+K_{j}^{\prime} \Lambda}+\sum_{j}^{N_{2}} \frac{\bar{K}_{j}^{21} \Lambda+2 \bar{K}_{j}^{21} \bar{K}_{j}^{22} \Lambda^{2}}{1+\bar{K}_{j}^{21} \Lambda+\bar{K}_{j}^{21} \bar{K}_{j}^{22} \Lambda^{2}}+\ldots+ \\
\sum_{j}^{N_{M}} \frac{\bar{K}_{j}^{M 1} \Lambda+\ldots+M \prod_{k}^{M} \bar{K}_{j}^{M k} \Lambda^{M}}{1+\bar{K}_{j}^{M 1} \Lambda+\ldots+\prod_{k}^{M} \bar{K}_{j}^{M k} \Lambda^{M}}
\end{array}
$$

Each fractional term in eq 16 involving polynomials of order $m$ corresponds to a polynomial (mplet) factor of the same order in the partition function in eq 15, i.e., to a quasigroup polynomial (these polynomials are obviously the denominators in the expression above). Since eq 16 is derived using only the basic thermodynamic arguments, it is the most general form of the total titration curve of a macromolecule with $N$ ligand-binding sites. Each term of order $m>1$ in eq 16 describes a binding curve with a cooperativity greater than 1 and less than or equal to $m$. The specific form of the many-body cooperativity suggested by eqs 15 and 16 does exist in real systems; an example will be presented later in this work, in the section on cooperative oxygen binding by hemoglobin.

Using the Decoupled Sites Representation for Data Fitting. The form of eq 16, although useful for theoretical analysis, may become cumbersome in practical applications, such as fitting of experimental data. One reason being that parameters $\left\{N_{1}\right.$, $\left.N_{2}, \ldots\right\}$ are a priori unknown and considering all possible combinations of them may be impractical, especially for large values of $N$. Also, using ratios of high-degree polynomials for fitting may not be the best choice from the point of view of numerical algorithms. To provide a simple and practical fitting procedure, we use the fact that roots of a polynomial always come in complex conjugate (CC) pairs: $z=\operatorname{Rez}+\operatorname{Im} z$ and $z^{*}$ $=R e z-\operatorname{Im} z$. Combining such pairs in eq 10, we break up the partition function into products over $(N-2 C)$ quasisites with real $K^{\prime}$ and $C$ pairs $\left(\operatorname{Re} K_{j}^{\prime} \pm \operatorname{Im} K_{j}^{\prime}\right)$ of $\mathrm{CC}$ ones

$$
\begin{aligned}
Z & =\prod_{i=1}^{N-2 C}\left[1+K_{i}^{\prime} \Lambda\right] \prod_{j=1}^{C}\left[1+K_{j}^{\prime} \Lambda\right]\left[1+K_{j}^{\prime *} \Lambda\right] \\
& =\prod_{i=1}^{N-2 C}\left[1+K_{i}^{\prime} \Lambda\right] \prod_{j=1}^{C}\left[1+2 \operatorname{Re} K_{j}^{\prime} \Lambda+\left|K_{j}^{\prime}\right|^{2} \Lambda^{2}\right]
\end{aligned}
$$

Applying eq 11, we obtain a purely real expression for the total average occupancy 


$$
\langle X\rangle=\sum_{i=1}^{N-2 C} \frac{K_{i}^{\prime} \Lambda}{\left(1+K_{i}^{\prime} \Lambda\right)}+\sum_{j=1}^{C} \frac{\alpha_{j} \Lambda+2 \gamma_{j} \Lambda^{2}}{1+\alpha_{j} \Lambda+\gamma_{j} \Lambda^{2}}
$$

where $K_{i}^{\prime}, \alpha_{j}$, and $\gamma_{j}$ are related to the roots $z$ of the binding polynomial eq 8 by

$$
\begin{gathered}
K_{i}^{\prime}=-z_{i}^{-1} \\
\alpha_{j}=2 \operatorname{Re} K_{j}^{\prime}=-\left(z_{j}^{-1}+z_{j}^{*-1}\right) \\
\gamma_{j}=\left(\operatorname{Re} K_{j}^{\prime}\right)^{2}+\left(\operatorname{Im} K_{j}^{\prime}\right)^{2}=z_{j}^{-1} z_{j}^{*-1}
\end{gathered}
$$

Just like eqs 16 or 5 , eq 18 is the most general form of the titration curve of a macromolecule with $N$ ligand-binding sites. Note that the Adair equation, ${ }^{4}$ often used to fit multiple ligand binding, is fully equivalent to eq 5 (for details, see the Supporting Information). The key difference of eq 18 from eq 5 or the Adair equation is that not only can $K_{i}^{\prime}, \alpha_{j}$, and $\gamma_{j}$ be treated as fitting parameters but the integer $C$ can as well. A similar but not identical approach was suggested before. ${ }^{26}$ Compared to eq 5, which is commonly used to fit ligand-binding curves, eq 18 has at least two advantages. First, the maximum power of polynomials involved in eq 18 is 2 , not $N$ as in eq 5 , which simplifies the fitting procedure and is less likely to result in a numerical instability. Second, eq 18 automatically provides some information about the degree of cooperativity of the ligand binding. As we shall see later, this information is contained in the parameter $C$, half the number of nonreal roots of the binding polynomial. In particular if $C=0$, eq 18 reduces to the sum of standard sigmoidal titration curves as described in our previous work. If $C>0$, the "quadratic" terms are present in eq 18 . Such a system can exhibit various degrees of cooperativity between 1 and $N$.

One has to keep in mind that some of the parameters $\alpha_{i}$ and $\gamma_{j}$ in eq 18 can be negative in which case a straightforward interpretation of these values as binding constants is not possible. Once, however, eq 18 has been fitted and the $K_{i}^{\prime}, \alpha_{i}, \gamma_{j}$, and $C$ have been determined, they can be used to obtain, via a straightforward procedure exemplified later in the work, a set of non-negative quasigroup constants specified in eq 16. The latter have physical meaning as explained above.

The described fitting procedure is as general as the Adair equation often used for fitting experimental data for cooperative binding. However, the mathematical expression (eq 18) to which the data are fitted is much simpler and less likely to cause numerical problems. In addition, the DSR parameter $\mathrm{C}$ provides additional information about the cooperativity of ligand binding.

A Quantitative Measure of Cooperativity. Experimental curves that describe ligand binding are sometimes very complex, and the widely used description in terms of Hill coefficient may be inadequate ${ }^{21}$ and even misleading. Consider for example a set of binding isotherms depicted in Figure 1, corresponding to a system of four binding sites. Although the visual appearance of the two curves is deceptively different, they both have the same mathematical form given by eq 18 with $C=1(N=4)$

$$
\langle X\rangle=\frac{K_{1}^{\prime} \Lambda}{\left(1+K_{1}^{\prime} \Lambda\right)}+\frac{K_{2}^{\prime} \Lambda}{\left(1+K_{2}^{\prime} \Lambda\right)}+\frac{\alpha \Lambda+2 \gamma \Lambda^{2}}{1+\alpha \Lambda+\gamma \Lambda^{2}}
$$

indicating the presence of cooperative binding. In the case of the dashed line, the domains of anti-cooperative (standard sigmoidal terms in eq 20) and cooperative ("quadratic" term) regimes nearly coincide, yielding a curve with a classical cooperative appearance. The Hill coefficient, defined as the slope of $\langle X\rangle /(N-\langle X\rangle)$ vs the chemical potential $\mu$ of the ligand at half saturation $\langle X\rangle=N / 2$, correctly indicates cooperative behavior, $n_{\mathrm{Hill}}>1$. One the other hand, $n_{\mathrm{Hill}}$ is much smaller than 1 for the solid curve and can be misinterpreted as a sign of strongly anti-cooperative behavior, which is obviously not the case. In fact, there are two well-separated domains in this case: cooperative, corresponding roughly to $\mu \gtrsim 0$, and anticooperative, where $\mu \lesssim 0$. Use of eq 18 to fit the experimental binding isotherms automatically eliminates these difficulties in interpretation; the best fit yields not only a set of $\left\{K_{1}, K_{2}, \ldots \alpha_{1}\right.$, $\left.\gamma_{1} \ldots\right\}$ but also $C$, which points to a degree of cooperativity possible in the system.

The integer $C$ can, as we shall see below, serve as a "global" measure of cooperativity, but if one goes further and identifies all of the constants and the quasigroups in eq 16, the degree of cooperativity can be quantified separately for each quasigroup. The example shown in Figure 1, representing a mixture of cooperative and anti-cooperative regimes, points to the necessity of such separation. A useful measure of cooperativity should indicate how sharply a quasigroup converts from a completely empty state to the completely filled one as ligand activity increases. This measure is clearly connected to the maximal slope of the ligand-binding curve.

In order to define the measure, we look at two extreme cases: a system that binds $N$ ligands non-cooperatively to $N$ identical noninteracting binding sites $\left(K=\mathrm{e}^{-\beta\left(G^{\circ}{ }{ }\right)}\right)$

$$
\begin{gathered}
Z_{\text {non-coop }}=(1+K \Lambda)^{N} \\
\left\langle X_{\text {non-coop }}\right\rangle=N \frac{\mathrm{e}^{-\beta\left(G^{\circ}{ }_{i}-\mu_{\mathrm{L}}\right)}}{1+\mathrm{e}^{-\beta\left(G^{\circ}-\mu_{\mathrm{L}}\right)}}
\end{gathered}
$$

and a system that binds $N$ ligands fully cooperatively, i.e., the system exists only in two states: all ligands bound or no ligands bound

$$
\begin{gathered}
Z_{\text {full-coop }}=1+K \Lambda^{N} \\
\left\langle X_{\text {full-coop }}\right\rangle=\frac{N \mathrm{e}^{-\beta\left(G^{\left.\mathrm{k}-N \mu_{\mathrm{L}}\right)}\right.}}{1+\mathrm{e}^{-\beta\left(G^{\left.\mathrm{k}-N \mu_{\mathrm{L}}\right)}\right.}}
\end{gathered}
$$

The maximum slope, i.e., the slope at the inflection point, is given in the non-cooperative case by

$$
\frac{\partial\left\langle X_{\text {non-coop }}\right\rangle}{\partial \mu_{\mathrm{L}}}=\frac{\beta N}{4}
$$

and in the cooperative case by

$$
\frac{\partial\left\langle X_{\text {full-coop }}\right\rangle}{\partial \mu_{\mathrm{L}}}=\frac{\beta N^{2}}{4}
$$

By consideration of eqs 23 and 24, we propose

$$
\Xi=\left.\frac{4}{\beta N} \frac{\partial\langle X\rangle}{\partial \mu_{\mathrm{L}}}\right|_{\text {at the inflection point }}
$$

as a new measure of cooperativity. For a system of two interacting sites, this measure is fully equivalent to the Hill coefficient (see Supporting Information for the formal proof), and so it can be interpreted as the number of ligands that bind simultaneously to one quasigroup. The definition eq 25 therefore provides a sensible measure for the degree of cooperativity of 


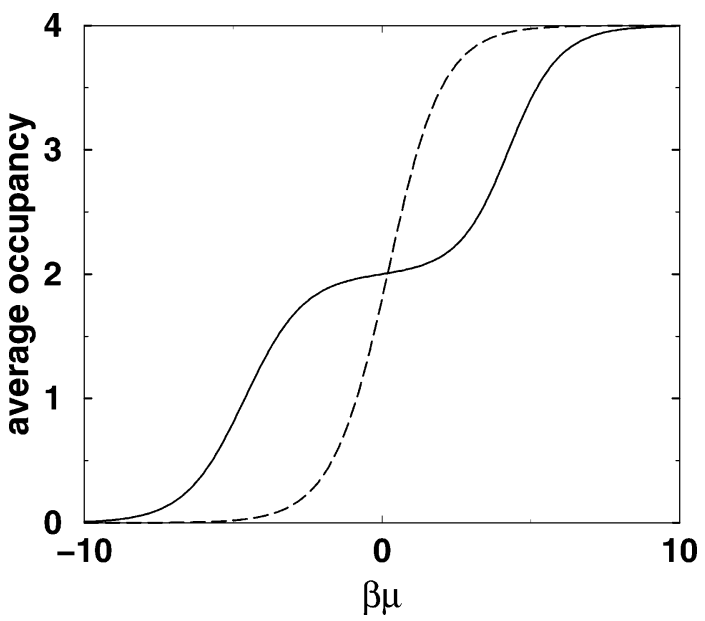

Figure 1. Two possible ligand-binding curves for a four-site system. The average total occupancy of the receptor $\langle X\rangle$ is depicted as a function of the ligand chemical potential (in units of $k_{\mathrm{B}} T, \beta \mu=\ln (\Lambda)$ ). Both curves have the same mathematical form of eq 20 with $\mathrm{C}=1$ indicating the presence of cooperative binding. Solid line, $K_{1}^{\prime}=100, K_{2}^{\prime}=100$, $\alpha=0.02, \gamma=0.0002$; dashed line, $K_{1}^{\prime}=1, K_{2}^{\prime}=1, \alpha=1, \gamma=0.5$. Note that the Hill coefficient $n_{\text {Hill }} \ll 1$ for the solid curve, which can be misinterpreted as the absence of cooperativity in the system.

a quasigroup $1<\Xi \leq N$. A detailed derivation of the cooperativity measure $\Xi$ and its relation to the Hill coefficient is given in the Supporting Information. As far as the integer number $C$ from eq 18 is concerned, clearly $C=0$ in the noncooperative case. In the fully cooperative case, $Z_{\text {full-coop }}$ from eq 22 has either zero (for even $N$ ) or one real root (for an odd $N$ ), and so $C=N / 2$ or $C=(N-1) / 2$, respectively.

The magnitude of $C$ characterizes the entire binding curve and serves as a "global" indicator of the possible degree of cooperativity. In this sense, it is similar to the Hill coefficient, although we will see later that $C$ is often a better indicator of what microscopic models are consistent with the experimental data. On the contrary, the measure $\Xi$ should not be applied to the total binding curve but instead to individual binding curves of each quasigroup, i.e., a degree of cooperativity $\Xi$ can be assigned to each quasigroup. This is one fundamental aspect in which it is different from the Hill coefficient. The cooperativity $\Xi$ of a quasigroup of order $m$ can adopt values between 1 and $m$

The values of the ligand chemical potential at which the titration curve is steep are the points where the receptor-ligand system is most sensitive to the changes in ligand activity. At such a point, a quasigroup may "respond" to the ligand and switch from one binding state to another, possibly transducing a signal. In the analysis of experimental data, it is therefore important to list not only the cooperativity measure $\Xi$, but also the chemical potential at the inflection point of the corresponding quasigroup binding curve.

\section{Results}

Receptor with Two Interacting Binding Sites. We now illustrate how the concepts developed above can be applied to a receptor with two binding sites. Despite its relative simplicity, the system exhibits rich behavior and serves as a good test model. It permits an exact analytical connection between the macroscopic binding constants on one hand and cooperativity signatures described above and physical parameters of the underlying microscopic model on the other. Since the development of a microscopic model is often the goal when a new ligandbinding system is investigated, it is important to understand the intricate connections between the two levels of description.

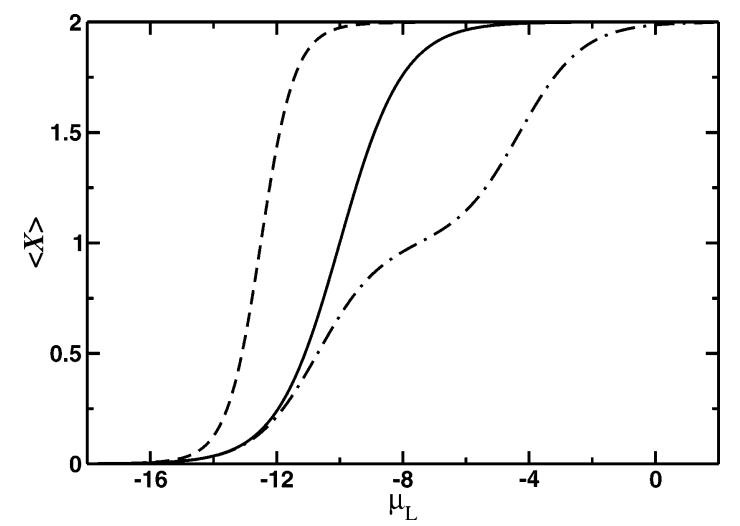

Figure 2. Total titration curves of a receptor with two identical ligands binding sites. The chemical potential $\mu_{\mathrm{L}}$ is given in units of $k_{B} T$. The energies for binding of the first or the second ligand to the receptor, $G_{1}^{\text {intr }}$ and $G_{2}^{\text {intr }}$, are the same $\left(-10 k_{\mathrm{B}} T\right)$. Different scenarios are illustrated: The two sites do not interact (solid curve, non-cooperative case); they interact repulsively with an energy of $5 k_{\mathrm{B}} T$ (dashed-dotted curve, anti-cooperative case); they interact attractively with an energy of $-5 k_{\mathrm{B}} T$ (dashed curve, cooperative case). The anti-cooperative case can also be interpreted as non-cooperative ligand binding to binding sites with different binding constants. Just from the titration alone, the differentiation between anti-cooperative and non-cooperative ligand binding cannot be made.

Receptor with Two Binding Sites and a Single Conformation. We consider a receptor that exists in a single conformation and has two ligand-binding sites. The ligands bind to the free receptor with binding energies of $G_{1}^{\text {intr }}$ and $G_{2}^{\text {intr }}$. When both sites are occupied, they interact with an energy $W$. The grand canonical partition function $Z$ of such a system is thus given by eq 26

$$
\begin{aligned}
& Z=1+A \Lambda+B \Lambda^{2} \\
& A=\mathrm{e}^{-\beta G_{1}{ }^{\text {intr }}}+\mathrm{e}^{-\beta G_{2}{ }^{\text {intr }}} \\
& B=\mathrm{e}^{-\beta\left(G_{1} \text { intr }_{\mathrm{G}_{2}}^{\text {intr }}+W\right)}
\end{aligned}
$$

where as before $\Lambda=\mathrm{e}^{\beta \mu_{\mathrm{L}}}$. The roots of $Z=0$ from eq 26 are real $(C=0$ in eq 18$)$ if

$$
W \geq-\frac{1}{\beta}\left(2 \ln \left(1+\mathrm{e}^{-\beta \Delta G}\right)-\ln 4\right)-\Delta G
$$

where $\Delta G=G_{2}^{\text {intr }}-G_{1}^{\text {intr }}$. The quasisite binding constants $K_{1}^{\prime}$ and $K_{2}^{\prime}$ are related to the parameters of the binding polynomial $Z$, eq 26. This relationship can be concisely expressed as $K_{1}^{\prime}+$ $K_{2}^{\prime}=A$ and $K_{1}^{\prime} K_{2}^{\prime}=B$. If eq 27 holds, the binding curve has a "two-step" shape as shown in Figure 2, which indicates anticooperative or non-cooperative binding. One typically observes these types of curves in $\mathrm{pH}$ titration experiments where the electrostatic repulsion between the protons results in $W>0$. When the inequality eq 27 is no longer true, the two roots of the binding polynomial are no longer real $(C=1 \mathrm{in} \mathrm{eq} \mathrm{18)}$ and the binding is described by a single "quadratic" term in eq 18 with $\alpha=A$ and $\gamma=B$. The corresponding titration curve is given by the dashed line in Figure 2 and represents cooperative binding. Perhaps somewhat counterintuitively, the mere presence of an attractive $(W<0)$ interaction between the binding sites is not sufficient to cause cooperativity. A more stringent condition is required. Figure 3 shows the regions of parameter space $(W, \Delta G)$ corresponding to cooperative and anti-cooperative regimes. If the binding affinities of the two sites are very different, the onset of cooperativity requires a strong attractive 

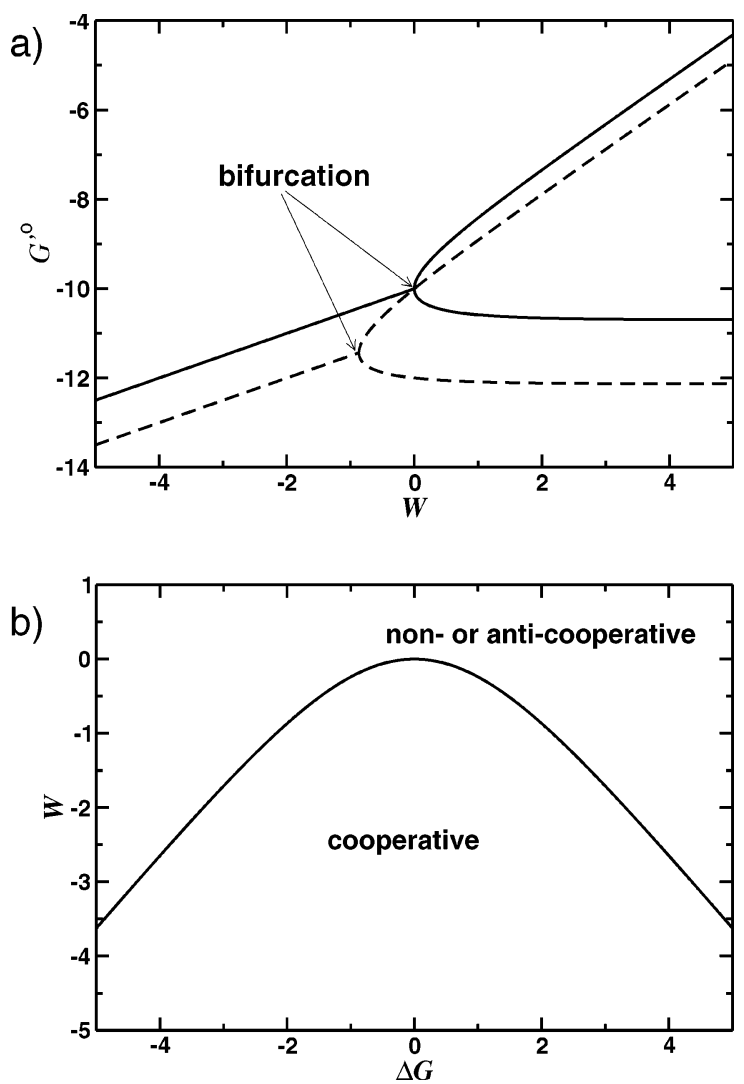

Figure 3. (a) Dependence of the quasisite binding constants on the interaction energy $W$ (in $k_{\mathrm{B}} T$ ) between the original (microscopic) sites. The $y$ axis gives the binding energy of a quasisite, which is obtained from $G_{i}^{\prime}=-\ln \operatorname{Re}\left(K_{i}^{\prime}\right)$. The intrinsic binding constants are $-10 k_{\mathrm{B}} T$ and $-10 k_{\mathrm{B}} T$ for the solid curve and $-10 k_{\mathrm{B}} T$ and $-12 k_{\mathrm{B}} T$ for the dashed curve. To the right of the bifurcation point, the two distinct roots of the binding polynomial are purely real, and the system can be decoupled into two independent quasisites, each characterized by its own binding constant $K_{i}^{\prime}$. To the left of the bifurcation point, the roots of the binding polynomial are complex, i.e., the binding is cooperative. This is when the two quasisites merge into one second-order quasigroup. Right at the bifurcation point the binding polynomial has 1 real root of multiplicity two, the binding is no longer cooperative. (b) Line separating the cooperative from the anti- and non-cooperative regimes. The line corresponds to the equality sign in eq 27 . All energies are given in $k_{\mathrm{B}} T$ units.

interaction between them. If the physical interaction between the sites is repulsive, $W>0$, the cooperative regime cannot be reached if the receptor can only exist in a single conformation. Another important conclusion that can be made is that the information contained in the total binding curve alone is not enough to distinguish between true anti-cooperativity and noncooperativity, since both are described by the same mathematical form. There is no way to decide just from the dash-dotted curve in Figure 2 whether it is produced by binding to independent sites $(W=0)$ having different intrinsic affinities to the ligand $(\Delta G \neq 0)$ or by identical $(\Delta G=0)$ interacting $(W>0)$ sites. Both scenarios belong to the same region in Figure $3 \mathrm{~b}$ specified by eq 27 . The separatrix line in Figure $3 \mathrm{~b}$ corresponds to the equality sign in eq 27 . It corresponds to a set of microscopic models $\left\{G_{1}^{\text {intr }}, G_{2}^{\text {intr }}, W\right\}$ with standard sigmoidal titration curves and equal quasisite binding constants $K_{1}^{\prime}=K_{2}^{\prime}=2 B / A$, as for instance the solid line in Figure 2. Only the point $\{W=0, \Delta G$ $=0\}$ on this line represents a system of two independent noninteracting sites that is truly non-cooperative. Since the twosite microscopic model is fully determined by three parameters $\left\{G_{1}^{\text {intr }}, G_{2}^{\text {intr }}, W\right\}$ and the macroscopic binding curve has only two parameters, the absence of a one-to-one mapping between the

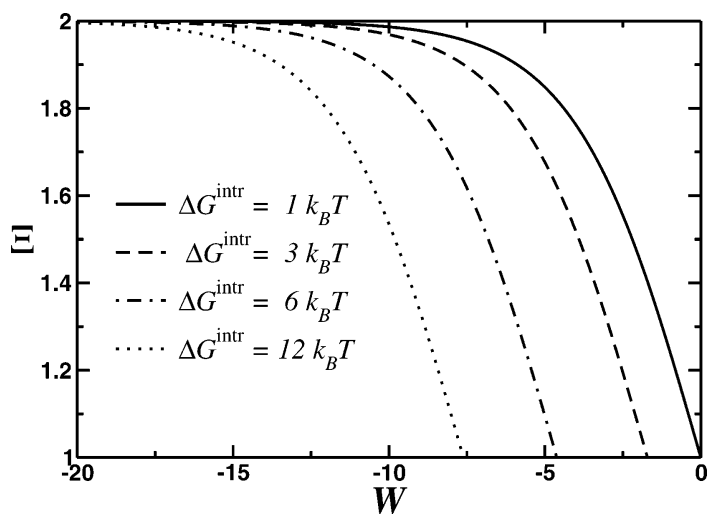

Figure 4. Dependence of the cooperativity measure $\Xi$ on the interaction strength $W$ between the original (microscopic) binding sites. The curves are drawn for several $\Delta G$ values representing the difference in ligandbinding affinity between the two sites. The sign of $\Delta G$ has no influences on the curve, i.e., the curves for $\Delta G$ and $-\Delta G$ are identical. Energy units are in $k_{\mathrm{B}} T$.

models is not surprising. Although a one-to-one mapping is also not possible for the cooperative regime, there is one important distinction from the anti-cooperative case: the binding curve cannot be reduced to a sum of two standard sigmoidal titration curves in the cooperative regime. Thus, cooperative binding curves cannot be interpreted as two independent sites. In the language of the DSR, the cooperative system can be described by one quasigroup capable of binding two ligands. In contrast, an anti-cooperative or non-cooperative system can be described by two quasisites each binding one ligand. The microscopic origin of this important distinction is in the fact that in the cooperative region there must exist an (effective) attractive interaction, $W<0$, between the ligands bound to the two sites.

Now we want to quantify the cooperativity of the system using the measure $\Xi$ from eq 25 . The $\Xi=1$ value separates the cooperative from the non-cooperative or anti-cooperative regimes, the last two scenarios being indistinguishable on the basis of a binding curve alone, as discussed above, and so it is only meaningful to assign $\Xi=1$ to both. In the qualitatively different cooperative regime $\Xi>1$, and Figure 4 illustrates the behavior of $\Xi$ as a function of $\Delta G$ and $W$. Note that the same value of $\Xi$ can correspond to different combinations of $\{\Delta G, W\}$. The cooperativity discussed in this section is brought about by attractive interaction between the ligands. Such an attraction can have various physical origins, such as for instance hydrophobic interactions or polar interactions. Attractive interaction is, however, unlikely between ligands which bear a net charge such as protons or metal ions, because of electrostatic repulsion. If cooperativity is found for such species, it is caused by other effects and not by a direct ligand-ligand attraction.

Receptor with Two Binding Sites and Two Conformations. We now consider a receptor with two binding sites. The receptor can exist in two conformations which differ in energy by $G^{\text {conf }}$. The binding energies of the ligands in the first conformation are $G_{1}^{\text {intr }}$ and $G_{2}^{\text {intr }}$ and in the second conformation $G_{\mathrm{A}}^{\text {intr }}$ and $G_{\mathrm{B}}^{\text {intr }}$. The interaction between the sites is $W_{12}$ in the first conformation and $W_{\mathrm{AB}}$ in the second. The energy diagram of the system is presented in Figure 5. Despite the simplicity of this model, its full parameter space is fairly large. A few simplifying assumptions help illustrate the key points. We assume that the difference in binding free energies of the two ligands is the same in the two conformations, $\Delta G_{\mathrm{AB}}^{\mathrm{intr}}=\Delta G_{12}^{\text {intr }}$ $=\Delta G$, and so is the interaction energy between the ligands in the two conformations, $W_{\mathrm{AB}}=W_{12}=W$. However, the individual ligand-binding free energies are assumed to be 


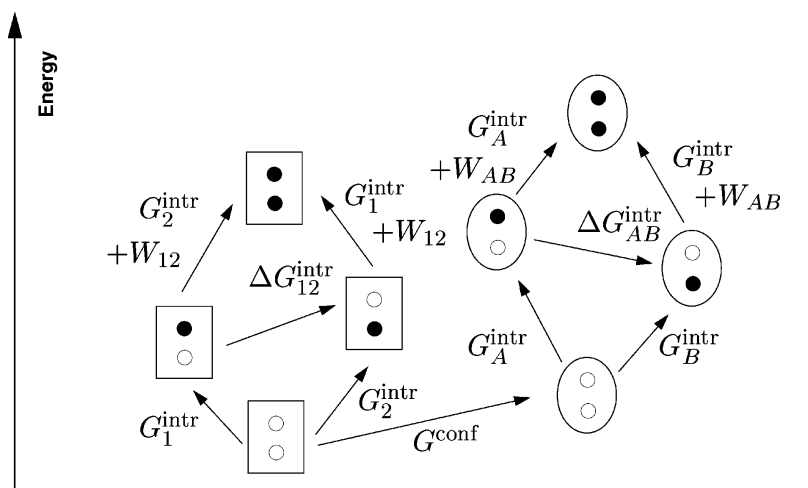

Figure 5. Energy diagram for a receptor with two interacting microscopic binding sites and two conformations. Empty and filled circles represent empty and ligand-bound sites.

different in the conformations. The difference is specified by $\Delta G_{\mathrm{A} 1}^{\text {intr }}=G_{\mathrm{A}}^{\text {intr }}-G_{1}^{\text {intr }}$. With these assumptions, the partition function is given by eq 26 with the coefficients $A$ and $B$ that are now functions of five free-energy parameters $G_{1}^{\text {intr }}, \Delta G, W$, $G^{\mathrm{conf}}$, and $\Delta G_{\mathrm{A} 1}^{\mathrm{intr}}$

$$
\begin{aligned}
& A= \\
& \frac{\mathrm{e}^{-\beta G_{1}^{\text {intr }}}+\mathrm{e}^{-\beta\left(G_{1}{ }^{\text {intr }}+\Delta G\right)}+\mathrm{e}^{-\beta\left(G_{1}{ }_{\text {intr }}+\Delta G_{\mathrm{Ai}}{ }^{\text {intr }}+G^{\text {conf }}\right)}+\mathrm{e}^{-\beta\left(G_{1}{ }^{\text {intr }}+\Delta G_{\mathrm{A}}{ }^{\text {int }}+\Delta G+G^{\text {conf }}\right)}}{1+\mathrm{e}^{-\beta G^{\text {conf }}}} \\
& B=\frac{\mathrm{e}^{-\beta\left(2 G_{1}{ }^{\text {int }}+\Delta G+W\right)}+\mathrm{e}^{-\beta\left(2\left(G_{1}^{\text {intr }}+\Delta G_{\mathrm{A}}{ }^{\text {int }}\right)+\Delta G+W+G^{\text {conf }}\right)}}{1+\mathrm{e}^{-\beta G^{\text {conf }}}}
\end{aligned}
$$

The first three parameters determine the energetics of a single conformation, and the last two pertain to the differences between the conformations.

First, consider $G^{\text {conf }}=0$, i.e., the free energy of the receptor is the same in both conformations without any ligands bound, but the affinities of the ligands to the different conformations differ. Similar to the receptor with only one conformation, different values of $\Delta G$ and $W$ determine whether the system behaves cooperatively or anti-cooperatively. However, the position of the line separating the non- and anti-cooperative regimes from the cooperative regime does depend on $\Delta G_{\mathrm{A} 1}^{\mathrm{intr}}$. In particular, the cooperative regime can now be reached even with repulsive interactions between the ligands (Figure 6a). The difference in ligand-binding free energy between the conformations acts as an effective attraction and can thus cause cooperative binding. The qualitative similarity between $W$ and $\Delta G_{\mathrm{A} 1}^{\text {intr }}$ in this respect does not, however, translate into their equivalence. While it is always possible to achieve cooperativity via a strong enough attractive $(W<0)$ interaction between the ligands, it is not the case with $\Delta G_{\mathrm{A} 1}^{\text {intr }}$ (Figure 6b). A relatively small repulsive interaction $W>k_{\mathrm{B}} T \ln 2$ can overwhelm even a hypothetically infinite $\Delta G_{\mathrm{A} 1}^{\mathrm{intr}}$ and cause anti-cooperative binding, as can be shown by simple algebra. If $W=0$, one can achieve cooperativity by increasing the value of $\Delta G_{\mathrm{A} 1}^{\mathrm{intr}}$, but the maximum cooperativity $\Xi$ that can be achieved in such a system is rather small, no larger than $\Xi<1.18$. Therefore, an observed value for $\Xi$ larger than 1.18 indicates that $\Delta G_{\mathrm{A} 1}^{\text {intr }}$ cannot be the only cause of cooperative ligand binding. Similar to the singleconformer case, $\Xi$ decreases with $\Delta G$, which means that $\Delta G_{\mathrm{A} 1}^{\text {intr }}$ alone is an even weaker cause of cooperative binding for nonequivalent sites, $\Delta G \neq 0$.

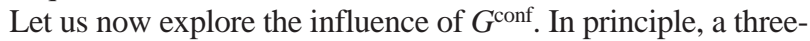
parameter space should be considered, as cooperativity is now a function of $W, \Delta G_{\mathrm{A} 1}^{\mathrm{intr}}$, and $G^{\mathrm{conf}}$. To make a useful graphical a)

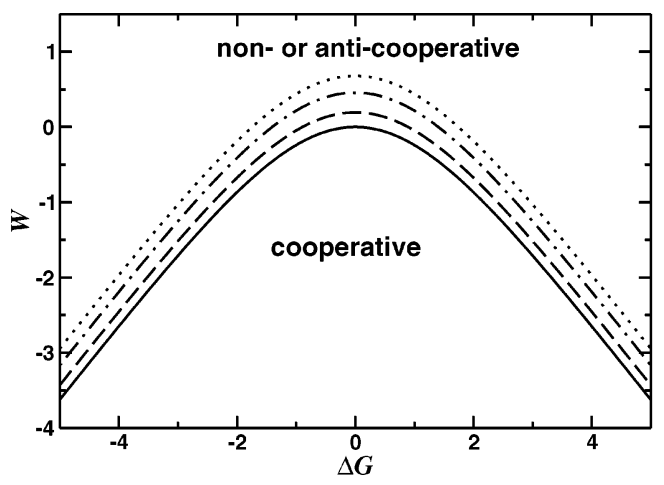

b)

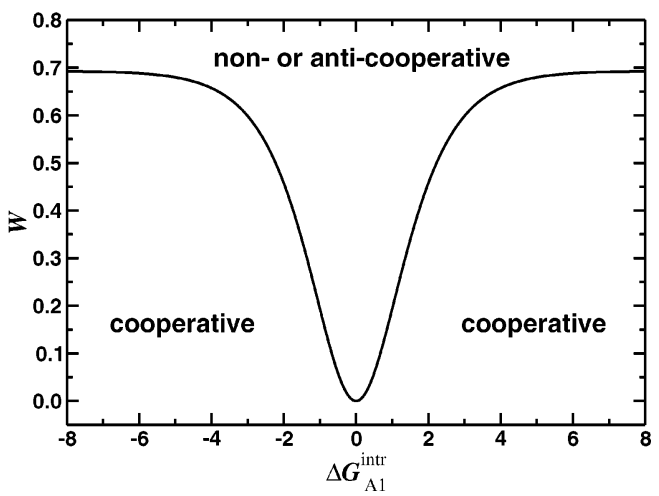

Figure 6. Lines separating the non- or anti-cooperative regime from the cooperative regime for a receptor that can exist in two conformations, each capable of binding two ligands. The energy difference between the conformations ( $G^{\text {conf }}$ ) is assumed to be zero. All energies are given in $k_{\mathrm{B}} T$ units. (a) Separatrix line for several $\Delta G_{\mathrm{A} 1}^{\text {intr }}$ (solid line, $-\Delta G_{\mathrm{A} 1}^{\text {intr }}=0 k_{\mathrm{B}} T$; dashed line, $-\Delta G_{\mathrm{A} 1}^{\mathrm{intr}}=1 k_{\mathrm{B}} T$; dashed-dotted line, $-\Delta G_{\mathrm{A} 1}^{\text {intr }}=2 k_{\mathrm{B}} T$; dotted line, $\left.-\Delta G_{\mathrm{A} 1}^{\text {intr }}=5 k_{\mathrm{B}} T\right)$. The spacing between the lines decreases with increasing $\Delta G_{\mathrm{A} 1}^{\mathrm{intr}}$. (b) The separatrix as a function of $\Delta G_{\mathrm{A} 1}^{\text {intr }}$ (for $\Delta G=0$ ).

representation of the observed trends, we limit the quantitative discussion to the case $W=0$ and focus on the $\left\{\Delta G_{\mathrm{A} 1}^{\mathrm{intr}}, G^{\mathrm{conf}}\right\}$ plane, Figure 7. The effect of nonzero $W$ was considered above and can be summarized as follows: attractive interaction $(W<$ 0 ) always enhances cooperative binding, and strong enough repulsion $(W>0)$ always leads to anti-cooperativity.

The cooperativity $\Xi$ as a function of $\Delta G_{\mathrm{A} 1}^{\mathrm{intr}}$ and $G^{\mathrm{conf}}$ is shown in parts a and b of Figure 7, respectively. While the mere existence of two different conformations, $G^{\text {conf }} \neq 0$, is not enough to cause cooperative binding (Figure 7c), it can enhance the cooperativity caused by other factors, such as attractive interactions between the ligands or difference in their binding affinity between the different conformations $\left(\Delta G_{\mathrm{A} 1}^{\mathrm{intr}} \neq 0\right)$.

Two Binding Sites for One Ligand Type Plus a Site for Another Type of Ligand. Now consider a receptor that can exist in a single conformation but has two ligand-binding sites for ligand of type $\mathrm{L}$ and one binding site for ligand of type $\mathrm{S}$. The interaction energy of the ligands of type $S$ with the ligands of type $\mathrm{L}$ is $W_{\mathrm{SL}}$. This interaction is assumed to be the same for both binding sites of type $\mathrm{L}$. The second-order binding polynomial can again be written in the form of eq 26 with

$$
\begin{aligned}
& A= \\
& \frac{\mathrm{e}^{-\beta\left(G_{1}{ }_{\text {intr }}+G_{\mathrm{S}}-\mu_{\mathrm{S}}+\mathrm{W}_{\mathrm{SL}}\right)}+\mathrm{e}^{-\beta\left(G_{1}{ }_{\text {intr }}+\Delta G+G_{\mathrm{S}}-\mu_{\mathrm{S}}+\mathrm{W}_{\mathrm{SL}}\right)}+\mathrm{e}^{-\beta G_{1}{ }_{\mathrm{intr}}}+\mathrm{e}^{-\beta G_{1} \text { intr }+\Delta G}}{1+\mathrm{e}^{-\beta\left(G_{\mathrm{S}}-\mu_{\mathrm{S}}\right)}} \\
& B=\frac{\mathrm{e}^{-\beta\left(2 G_{1}^{\text {int }}+\Delta G+\mathrm{W}_{\mathrm{SL}}\right)}+\mathrm{e}^{-\beta\left(2 G_{1}{ }_{\text {intr }}+\Delta G+G_{\mathrm{S}}-\mu_{\mathrm{S}}+2 \mathrm{~W}_{\mathrm{SL}}\right)}}{1+\mathrm{e}^{-\beta\left(G_{\mathrm{S}}-\mu_{\mathrm{S}}\right)}}
\end{aligned}
$$


a)

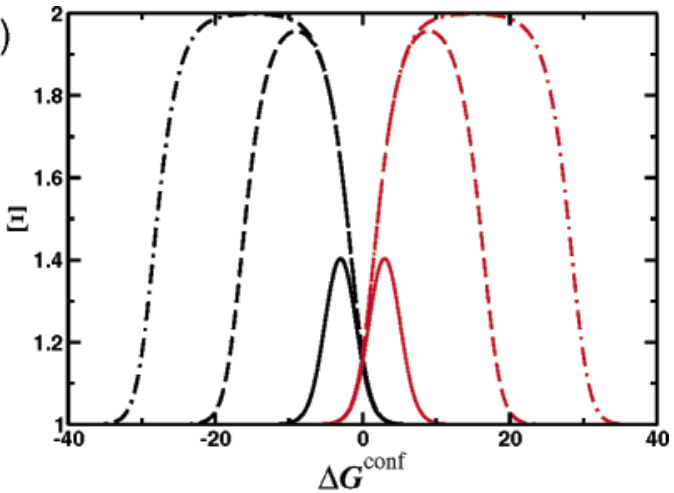

b)
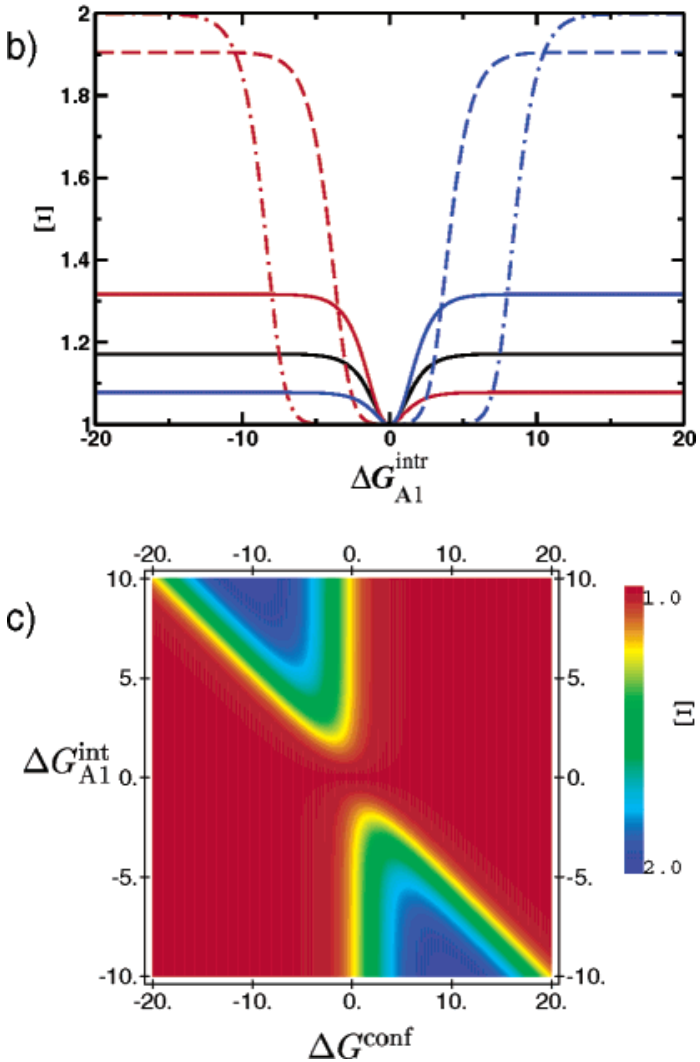

Figure 7. Cooperativity in a two-site system that can exist in two conformations. The cooperativity is measured by $\Xi$ introduced in eq 25 . The conformational energy difference between the two conformations is $G^{\text {conf }}$, and the difference in the intrinsic binding constants is $\Delta G_{\mathrm{A} 1}^{\text {intr }}$. All energies are given in $k_{\mathrm{B}} T$, and $W$ is set to zero. (a) $\Xi$ vs $G^{\text {conf }}$ for different $\Delta G_{\mathrm{A} 1}^{\text {intr }}$ : solid black, $3 k_{\mathrm{B}} T$; dashed black, $9 k_{\mathrm{B}} T$; dashed-dotted black, $15 k_{\mathrm{B}} T$; solid red, $-3 k_{\mathrm{B}} T$; dashed red, $-9 k_{\mathrm{B}} T$;

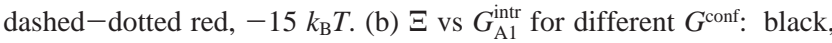
$0 k_{\mathrm{B}} T$; solid red, $1 k_{\mathrm{B}} T$; dashed red, $6 k_{\mathrm{B}} T$; dashed-dotted red, $15 k_{\mathrm{B}} T$; solid blue, $-1 k_{\mathrm{B}} T$; dashed blue, $-6 k_{\mathrm{B}} T$; dashed-dotted blue, -15 $k_{\mathrm{B}} T$. (c) Two-dimensional representation of $\Xi$ (color code) as a function of $G_{\mathrm{A} 1}^{\text {intr }}$ and $G^{\mathrm{conf}}$.

where $G_{\mathrm{S}}$ and $\mu_{\mathrm{S}}$ are the binding affinity and chemical potential of the ligand of type $S$ and the other variables have the same meaning as in eq 28 and Figure 5. Equation 29 becomes fully equivalent to eq 28 upon substitution of $W_{\mathrm{S}}=\Delta G_{\mathrm{A} 1}^{\mathrm{intr}}$ and $G_{\mathrm{S}}-$ $u_{\mathrm{S}}=G^{\mathrm{conf}}$. This substitution maps the problem at hand onto the already discussed problem of two ligands and a receptor with two conformations. We therefore do not discuss it further here and refer the reader to the previous section.

The case of nonattractive interaction between ligands of one type and attraction between different types is of special interest, as it is found in many experimental systems such as coupling between protonation and reduction $\left(\mathrm{H}^{+}\right.$and $\left.\mathrm{e}^{-}\right)$. The cooper- ativity for binding a ligand of one type can be caused by the binding of a ligand of a different type, in the same way as it can be induced by conformational dependence of binding.

One should also keep in mind that other combinations of the above sketched scenarios are possible. For instance, it is possible that the ligands of type $\mathrm{L}$ and type $\mathrm{S}$ do not interact but the receptor exists in two conformations. One conformation binds the ligand of type $\mathrm{S}$ with a higher affinity. In this conformation, the ligands of type $\mathrm{L}$ have a higher affinity and thus could also bind cooperatively to the receptor. This scenario is probably realized for the Bohr effect of hemoglobin.

Cooperative binding can be realized via many different microscopic mechanisms. Simple binding studies, although very important and helpful, cannot provide a understanding of the binding mechanism at a microscopic level and need to be complemented by other studies.

The equivalence between the chemical potential $\mu_{\mathrm{S}}$ and the conformational energy $G^{\text {conf }}$ is biologically of great interest. While conformational energy of a receptor can only be changed by a mutation and thus is not a useful regulatory mechanism at the level of a single organism, it is easily possible to change the chemical potential of a ligand and thus change the degree of cooperativity of the receptor by varying the ambient conditions. For example, calcium concentration in cells regulates many physiological processes, e.g., muscle contraction.

Receptor With More Than Two Binding Sites. While for $N>2$ the algebra becomes more intricate and is best treated on a case-by-case basis, ${ }^{7,25}$ several general conclusions are still worth mentioning. As in the two-site case, a fully factorizable polynomial in the form of eq 8 with all real $K_{i}^{\prime}$ values $(C=0$ in eq 18) represents a completely non-cooperative or anticooperative case. The total binding curve is a sum of $N$ standard sigmoidal titration curves.

For a system that is not fully factorizable in the above sense, and has the highest irreducible polynomial of the order $M$, the maximum cooperativity $\Xi$ cannot exceed $M$, the maximum possible cooperativity of quasigroup of order $M$. Note that $M$ $\leq 2 C$ for even $N$ and that $M \leq 2 C+1$ for odd $N$. No matter how large $M$ is, even if $M=N$, the cooperativity in the system can be as low as 1, that is, $\Xi$ can only be infinitesimally larger than unity. This property can be seen from considering a binding polynomial of the form $Z=(1+K \Lambda)^{N}+\epsilon, \epsilon>0$, whose roots lie on a circle of radius $K^{-1} \epsilon^{1 / n}$ centered at $\left(-K^{-1}, 0\right)$ in the complex plane. For small $\epsilon$, the above polynomial corresponds to a system of nearly noninteracting identical sites, each characterized by $\Xi \approx 1$.

The most important general conclusions made in the previous section for a two-site system remain valid in the $N>2$ case and are listed below. It is impossible to infer, from apparently non-cooperative binding curves alone, whether the microscopic interactions between the binding sites exist, or if the system is composed of truly independent sites. For cooperative binding to occur, an effective microscopic interaction between the sites must be present. This interaction may have various origins, such as difference in ligand-binding constants in different conformations of the receptor, binding of another type of ligand or real attractive interaction between the ligands.

Hemoglobin: A Test Case. Oxygen binding to hemoglobin is probably the most studied of cooperative ligand-binding phenomena. As such, it presents a perfect test case for a novel approach such as DSR.

Historically, our understanding of oxygen binding to hemoglobin was based on two types of models that can be broadly defined as "phenomenological" or macroscopic and "structural" 
TABLE 1: DSR Fitting Parameters of Experimental Oxygen Binding Curves of Carp Hemoglobin at Several pH Values ${ }^{a}$

\begin{tabular}{|c|c|c|c|c|c|c|c|c|c|}
\hline \multirow[b]{2}{*}{$\mathrm{pH}$} & \multicolumn{7}{|c|}{ DSR fitting parameters, eq 18} & \multirow[b]{2}{*}{ MWC } & \multirow[b]{2}{*}{$n_{\text {Hill }}^{\max }$} \\
\hline & $K_{1}^{\prime}$ & $K_{2}^{\prime}$ & $\alpha_{1}$ & $\gamma_{1}$ & $\alpha_{2}$ & $\gamma_{2}$ & $C$ & & \\
\hline 6.25 & 0.00867 & 0.042 & 0.00288 & 0.000205 & & & 1 & no & 1.34 \\
\hline 6.61 & 0.0420 & 0.0797 & -0.0190 & 0.00112 & & & 1 & no & 1.93 \\
\hline 6.92 & & & 0.1414 & 0.00771 & -0.0586 & 0.00461 & 2 & yes & 2.33 \\
\hline 7.20 & & & 0.326 & 0.0287 & -0.0760 & 0.0256 & 2 & yes & 1.82 \\
\hline 7.35 & & & 0.540 & 0.140 & -0.01750 & 0.064 & 2 & yes & 1.62 \\
\hline 7.65 & & & 1.164 & 0.492 & 0.1302 & 0.211 & 2 & yes & 1.42 \\
\hline 7.83 & & & 1.800 & 1.33 & 0.322 & 0.344 & 2 & yes & 1.32 \\
\hline 8.37 & 1.068 & 2.735 & 0.192 & 0.349 & & & 1 & no & 1.36 \\
\hline 9.11 & 3.840 & 4.92 & 1.276 & 0.925 & & & 1 & no & 1.06 \\
\hline
\end{tabular}

${ }^{a}$ In the DSR fitting procedure based on eq $18, C$ is treated as a discrete variable; $C=0,1$, or 2 . The value that provides the best global fit to experiment is listed. The next-to-last column indicates whether the particular binding isotherm can be described by a two-state MWC model. Experimental data used in the fit, and the Hill coefficient for each binding curve are from ref 28.

TABLE 2: Quasigroup Binding Constants of the DSR Model in the Form of Equation 16 that Describe the Binding of Oxygen to Carp Hemoglobin at Different $\mathbf{p H}$ Values $^{a}$

\begin{tabular}{|c|c|c|c|c|c|c|c|}
\hline $\mathrm{pH}$ & & quasig! & nstants & & $\mathbf{m}$ & $\Xi$ & $k_{\mathrm{B}} T \ln \left[\mathrm{O}_{2}\right]$ \\
\hline \multirow[t]{3}{*}{6.25} & $K_{1}^{\prime}=0.0087$ & & & & 1 & 1.00 & 4.74 \\
\hline & $\bar{K}_{1}^{21}=0.0029$ & $\bar{K}_{1}^{22}=0.0711$ & & & 2 & 1.82 & 4.24 \\
\hline & $K_{2}^{\prime}=0.0428$ & & & & 1 & 1.00 & 3.15 \\
\hline \multirow[t]{2}{*}{6.61} & $\bar{K}_{1}^{31}=0.0231$ & $\bar{K}_{1}^{32}=0.0139$ & $\bar{K}_{1}^{33}=0.1474$ & & 3 & 1.64 & 3.39 \\
\hline & $K_{1}^{\prime}=0.0797$ & & & & 1 & 1.00 & 2.54 \\
\hline 6.92 & $\bar{K}_{1}^{41}=0.0828$ & $\bar{K}_{1}^{42}=0.0486$ & $\bar{K}_{1}^{43}=0.0497$ & $\bar{K}_{1}^{44}=0.1780$ & 4 & 2.30 & 2.30 \\
\hline 7.20 & $\bar{K}_{1}^{41}=0.2504$ & $\bar{K}_{1}^{42}=0.1179$ & $\bar{K}_{1}^{43}=0.2100$ & $\bar{K}_{1}^{44}=0.1190$ & 4 & 1.82 & 1.83 \\
\hline 7.35 & $\bar{K}_{1}^{41}=0.5240$ & $\bar{K}_{1}^{42}=0.3735$ & $\bar{K}_{1}^{43}=0.1653$ & $\bar{K}_{1}^{44}=0.2800$ & 4 & 1.59 & 1.31 \\
\hline \multirow[t]{2}{*}{7.65} & $\bar{K}_{1}^{21}=0.1303$ & $\bar{K}_{1}^{22}=1.6193$ & & & 2 & 1.75 & 0.78 \\
\hline & $\bar{K}_{1}^{21}=1.1657$ & $\bar{K}_{1}^{22}=0.4224$ & & & 2 & 1.09 & 0.36 \\
\hline \multirow[t]{2}{*}{7.83} & $\bar{K}_{1}^{21}=0.3228$ & $\bar{K}_{1}^{22}=1.0659$ & & & 2 & 1.57 & 0.53 \\
\hline & $\bar{K}_{1}^{21}=1.8012$ & $\bar{K}_{1}^{22}=0.7421$ & & & 2 & 1.12 & -0.14 \\
\hline \multirow[t]{3}{*}{8.37} & $K_{1}^{\prime}=1.0684$ & & & & 1 & 1.00 & -0.06 \\
\hline & $K_{2}^{\prime}=2.7351$ & & & & 1 & 1.00 & -1.01 \\
\hline & $\bar{K}_{1}^{21}=0.1924$ & $\bar{K}_{1}^{22}=1.8172$ & & & 2 & 1.72 & 0.53 \\
\hline \multirow[t]{3}{*}{9.11} & $K_{1}^{\prime}=3.8405$ & & & & 1 & 1.00 & -1.59 \\
\hline & $K_{2}^{\prime}=4.9218$ & & & & 1 & 1.00 & -1.35 \\
\hline & $\bar{K}_{1}^{21}=1.2777$ & $\bar{K}_{1}^{21}=0.7248$ & & & 2 & 1.20 & 0.04 \\
\hline
\end{tabular}

${ }^{a}$ All binding constants are real positive numbers. Also listed are the order $\mathbf{m}$ and cooperativity measure $\Xi$ for each quasigroup as well as the ligand chemical potential (last column) at which the titration curve has the steepest slope.

or microscopic. A pure example of the first type of model is the Adair description. ${ }^{4}$ The chemical mechanism proposed by Perutz $^{27}$ is an example of the second approach. The MWC model $^{5}$ is between the two approaches; it is a phenomenological model based on some structure-based assumptions that limit the model's conformational space ("symmetry model"). In this respect, the DSR approach based on eq 18 clearly belongs to the macroscopic models. The strength of such a very general method is in its applicability to a very broad class of systems, regardless of underlying molecular mechanisms. Its advantage is in the ability to provide guidance for more specific model building.

The oxygen binding to hemoglobin from carp is a particularly interesting test case. It is characterized by a remarkable $\mathrm{pH}$ dependence, which at some $\mathrm{pH}$ values cannot be fitted to the MWC model. Here we use the DSR model (eq 18) to fit the experimental data obtained by Chien and $\mathrm{Mayo}^{28}$ for the oxygen binding to carp hemoglobin at different $\mathrm{pH}$. The fitted parameters are listed in Table 1. These parameters fit the data very well, which is not surprising as the framework of the DSR is as general as the Adair equation (see Supporting Information for details); the latter was used in ref 28 to fit the same sets of experimental data. However, fitting to eq 18 is less likely to present numerical problems, since it uses polynomials of degree no larger than two. More importantly, compared to the Adair equation, the DSR procedure provides more information about the cooperativity of binding. Namely, the integer parameter $C$ obtained as a result of the best-fit procedure is an indicator of the types of cooperative couplings possible in the system. The general restrictive conclusions that can be made from the $C$ values can be used in building the underlying microscopic model. In particular, if $C \neq 0$, one can assert that there must exist an effective interaction between the sites at the microscopic level. If $C=1$, it is possible that the underlying microscopic model has two (out of four) sites that do not interact with each other, while for $C=2$, this is impossible. The last column of Table 1 indicates whether MWC model is an admissible description of the binding isotherm at the given $\mathrm{pH}$. While there appears to be no clear correlation between the applicability of the two-state MWC model and the Hill coefficient $n_{\mathrm{Hill}}$, there is a clear one-to-one correspondence with the $C$ values; for $C$ $=2$ the model is admissible, and for $C<2$, it is not. The proof of this fact is based on the analysis ${ }^{29}$ of zeros of the binding polynomial corresponding to the (two-state) MWC model; it was shown $n^{29,30}$ that the latter always has two pairs of complex roots. Note that no complex numbers are used in eq 18 and that the global indicator of cooperativity $C$ is automatically obtained as a fitting parameter. In practice, one performs a least- 
squares procedure based on eq 18 with different values of $C=$ 0,1 , and $N / 2$ (or $(N-1) / 2$ for an odd $N$ ) and chooses $C$ that corresponds to smallest $\chi^{2}$. It may so happen that two or more $\chi^{2}$ values corresponding to consecutive values of $C$ s come out to be identical, within the accuracy of the numerical procedure. In this case, one should check if any of the denominators in eq 16 allow further factoring into a product of two linear terms with non-negative coefficients $K^{\prime}$, i.e., $\left(1+\alpha_{i} \Lambda+\gamma_{i} \Lambda^{2}\right)=(1$ $\left.+K_{1}^{\prime} \Lambda\right)\left(1+K_{2}^{\prime} \Lambda\right)$, in which case the corresponding fraction can be reduced to a sum of two fractions, each being in the standard sigmoidal form. The value of $C$ should then be reduced by 1 . The factoring is possible if $\alpha_{i}^{2}-4 \gamma_{i} \geq 0$, and so one tests each pair $\left\{\alpha_{i}, \gamma_{i}\right\}$ for the above inequality and reduces $C$ by 1 for each pair for which it holds. For example, for carp hemoglobin at $\mathrm{pH}=6.25, \chi^{2}$ corresponding to $C=1$ and $C=$ 2 are the same, and we find that the above condition is satisfied for one pair of $\left\{\alpha_{i}, \gamma_{i}\right\}$, and so $C$ is 1 , not 2 , for this data set, Table 1.

It is also possible that some of the fitting parameters $\alpha$ are negative, e.g., at $\mathrm{pH}=6.61$, which means that quasigroups of an order larger than two are present in the DSR decomposition eq 18. To see this, we combine (add) the term with $\alpha<0$ with other terms in the decomposition until we obtain fractions in which the denominator polynomials have only positive coefficients, i.e., become irreducible. In other words, we recast the binding curve in the form of eq 16 . It was shown before ${ }^{31}$ that the decomposition into irreducible polynomials is unique for $N$ $\leq 5$. Each irreducible polynomial in the denominator is the irreducible sub-polynomial in the partition function eq 15 and as such corresponds to a quasigroup introduced earlier in this study. The real positive binding constants that characterize quasigroups for carp hemoglobin are listed in Table 2 along with the order of each quasigroup, i.e., the degree $M$ of the corresponding binding sub-polynomial. Also listed in Table 2 is the cooperativity measure $\Xi$ for each quasigroup and the ligand chemical potential at which the slope of the quasigroup binding curve reaches its maximum.

We now analyze the results presented in Tables 1 and 2 in some detail. From the values of either $C$ or $\Xi$, it follows that carp hemoglobin binds oxygen cooperatively at all $\mathrm{pH}$ values considered here. At $\mathrm{pH}=6.25,8.37$, and 9.11, the cooperativity is the lowest, and the binding curve can be decomposed into a second-order quasigroup plus two single quasisites (i.e., quasigroups of order 1$)$. The combined "order" $(2+1+1)=4$ equals the number of binding sites, as expected. The cooperativity measure $\Xi$ for a quasigroup is always larger than 1.0, while it is always exactly 1.0 for a single quasisite. An example of the binding curve with the above quasigroup decomposition is shown in Figure 8a.

At $\mathrm{pH}=6.61$, the total binding curve can be decomposed into a third-order quasigroup plus one quasisite, i.e., $(3+1)=$ 4. The fitting to eq 18 leads to $C=1$ in this case, but since the fitting parameter $\alpha$ is negative, the above interpretation requires combining the terms in eq 18 to obtain the form of eq 16 . In practice, one simply forms products of the denominators, since these correspond to binding sub-polynomials. For $\mathrm{pH}=6.61$, it works out as

$$
\begin{aligned}
& \left(1+\alpha_{1} \Lambda+\gamma_{1} \Lambda^{2}\right)\left(1+K_{1}^{\prime} \Lambda\right)= \\
& 1+0.0607 \Lambda-0.0003943 \Lambda^{2}+0.000089264 \Lambda^{3} \\
& \left(1+\alpha_{1} \Lambda+\gamma_{1} \Lambda^{2}\right)\left(1+K_{2}^{\prime} \Lambda\right)= \\
& 1+0.023 \Lambda+0.000322 \Lambda^{2}+0.00004704 \Lambda^{3}
\end{aligned}
$$
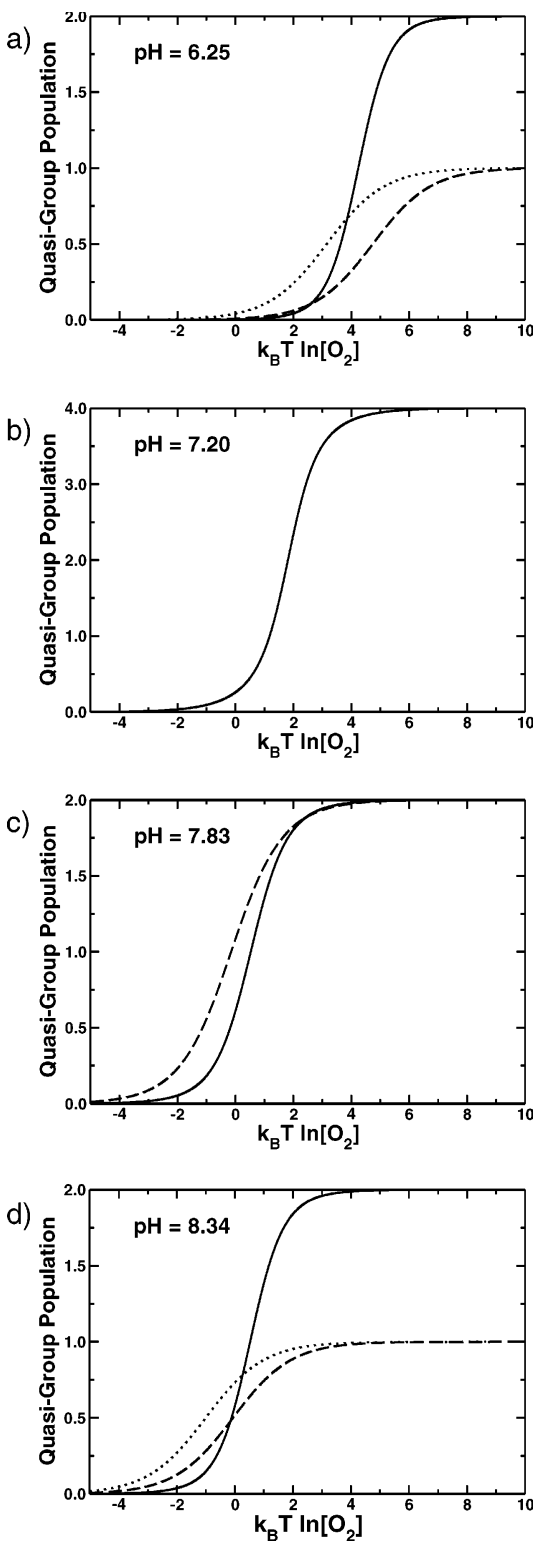

Figure 8. Decomposition of the overall binding isotherm of carp hemoglobin into titration curves corresponding to the decoupled quasigroups identified by the DSR analysis. (a) At $\mathrm{pH}=6.25$, the total titration curve can be interpreted as a combination of the second-order quasigroup (solid line) and two independent quasisite sites (dashed and dotted lines). (b) At $\mathrm{pH}=7.20$, the titration curve is that of a fourthorder quasigroup; no decoupling is possible. (c) At $\mathrm{pH}=7.83$, the DSR identifies a pair second-order quasigroups characterized by different cooperativity measures $\Xi$ at different ligand concentrations. (d) At $\mathrm{pH}=9.94$, the situation is similar to a, except that the binding occurs in a different range of the ligand concentration.

Only the second combination yields a polynomial that can be a binding polynomial. The combination of the parameters that yields all real and positive quasigroup binding constants is unique, as is the decomposition of the binding polynomial into irreducible sub-polynomials. The quasigroup decomposition contains, in itself, information that may be useful in constructing the underlying microscopic model. In the above example, $(1+$ $3)$, the corresponding microscopic model cannot consist of completely independent sites $(1+1+1+1)$, or a pair of interacting sites plus two independent sites $(2+1+1)$, or two pairs of sites with no interaction outside of each pair $(2+2)$, or sites interacting in such a way that their binding polynomial cannot be factored into lower-order sub-polynomials (4). 
At $\mathrm{pH}=6.92,7.20$, and 7.35, each binding curve is described by a fourth-order quasigroup, (4), consisting of four sites that cannot be decoupled via a linear transformation (such as the DSR). In this case, one of the fitting parameters $\alpha$ in Table 1 is again negative, and so the product of the two quadratic subpolynomials in the denominators will yield a fourth order (irreducible) binding polynomial. For each of these three $\mathrm{pH}$ values, only one cooperativity measure $\Xi$ can be given because there is only one quasigroup. An example of such a curve is depicted in Figure $8 b$.

At $\mathrm{pH}=7.65$ and 7.83 , the binding curve can be decomposed into two second-order quasigroups $(2+2)$. Both quasigroups do not interact with each other and have different ligand sensing abilities. For illustration, we focus on the binding curves at $\mathrm{pH}$ 7.83, which is depicted in Figure 8c. At this $\mathrm{pH}$, we have two quasigroups with different binding behavior. The first group (dashed line in Figure 8c) has a higher affinity than the other one, i.e., it binds the ligand at a lower value of the ligand chemical potential. The cooperativity of this quasigroup is relatively low, i.e., it goes from the free to the completely filled state via a relatively smooth transition. The second quasigroup (solid line in Figure 8c) has a lower affinity, but its cooperativity is higher, i.e., it changes from the free to the completely filled state via a relatively sharp transition. This example shows that it is possible to have two different quasigroups that exhibit different cooperative behavior. In principle, even a larger affinity difference between the quasigroups is possible. Such a behavior may be important for sensor proteins that need to sense different levels of ligand concentration, as is believed to be the case for several calcium-binding proteins. ${ }^{32}$ In the last example, one can imagine two quasigroups sensing different levels of calcium concentration. Moreover, since the quasigroup cooperativity can differ, they can switch, with different sensitivity, between the active and the inactive forms as the ligand concentration changes.

\section{Conclusions}

In this paper, we have extended the framework of the DSR, previously developed in the context of $\mathrm{pH}$ titration, to include cooperative ligand binding. To treat both cooperative and anticooperative binding within the same framework, we have reformulated the DSR in terms of physically interpretable, purely real and positive, binding constants of quasigroups. The latter is a generalization of the quasisite concept for the case when two or more sites cannot be viewed as noninteracting due to the strength of cooperative interactions between them. A quasigroup is characterized by a set of binding constants whose number equals the order of the quasigroup, the degree of the corresponding irreducible binding polynomial. We have also extended the DSR framework to include multiple conformers and more than one types of ligand.

A particularly simple form of the DSR is proposed that is well-suited for least-squares fitting of experimental binding curves. Along with the DSR decomposition constants, the fitting procedure automatically produces an integer parameter indicative of the degrees of cooperativity possible in the system.

We have used an analytically tractable model of a receptor with two binding sites to explore various cooperative regimes possible in the system, as well as the relationship between the macroscopic description in terms of binding isotherms and the parameters of the underlying microscopic model. We show that without a microscopic model it is not possible to make a meaningful distinction between non-cooperative and anti- cooperative regimes, as these can result in identical binding curves. In general, different microscopic models can lead to the same macroscopic behavior. Thus information in addition to binding constants is required to differentiate between different microscopic models. However, one can differentiate from the binding constants and from the binding curves between cooperative and anti-cooperative ligand binding. We use the twosite example to explore possible microscopic origins of (positive) cooperativity, which include effective attractive interactions, changes in the receptor's conformation upon binding, and binding of another type of ligand that influences the ligand affinity. Interestingly, the model describing the cooperativity of binding caused by conformational changes and the one caused by binding of another type of ligand are mathematically equivalent. This finding is of significant biological interest, because either of the two mechanisms for adjusting cooperativity can be used in biological systems depending on particular circumstances. For example, it is generally very hard, on short time-scales and under normal physiological conditions, to change the conformational energy of a molecule. Under these conditions it is, however, relatively easy to change the concentration of another type of ligand, an effector, and thus change the cooperativity as needed. The adjustment of the conformational energy may come into play on longer time scales, e.g., during embryogenesis. There, chemical modifications, expression of other genes for a given receptor, or even different splicing schemes for the receptor may lead to changes in the conformational energy and so regulate the cooperativity. On even longer, evolutionary-relevant time scales, mutations may lead to changes in cooperative patterns on the molecular level, thus allowing the species to adapt to the changes in the ecological environment.

We have also explored the usefulness of the new framework as applied to the well-known test case of a highly cooperative phenomenon, oxygen binding to hemoglobin. Using available experimental data on oxygen binding to carp hemoglobin at different $\mathrm{pH}$, we have employed the DSR to identify several types of cooperative behavior possible in the system. Each type corresponds to a different decomposition of the total titration curve into quasigroup components. These decompositions indicate the limiting behavior of the system upon site decoupling and can be used in building an underlying microscopic model. The integer parameter $C$, resulting from the DSR least-squaresfit procedure of the experimental data, provides an indication whether of not certain microscopic models (such as the twostate MWC model) are applicable to the given data set.

The new measure of cooperativity $\Xi$ introduced in this work describes how sensitive a quasigroup is to the change of the ligand concentration. Each of the quasigroups found in the carp hemoglobin system titrates at a slightly different $\mathrm{pH}$ and is characterized by a different value of $\Xi$. As a possible practical application of the above phenomenon, we note that a sensor molecule designed to have similar behavior would be particularly sensitive to changes in the ligand concentration not just at one but at several values of the ligand concentration, one per each quasigroup. This type of mechanism may be realized by calcium-binding proteins such as $\$ 100$, which need to react differently at different levels of calcium concentration.

The extended DSR framework, which now includes both cooperative and anti-cooperative binding on an equal footing, is not an approximation and is based on very general thermodynamic arguments. It provides insights into ligand-binding phenomena and can be useful in fitting experimental data and in selecting the underlying microscopic models. 
The software developed to perform the data analysis described in this work is freely available at www.cs.vt.edu/ onufriev.

Acknowledgment. G.M.U. was supported by an EmmyNoether Grant of the Deutsche Forschungsgemeinschaft (UL 174/2). We thank Elisa Bombarda and Layne Watson for helpful discussions, David Case for support and encouragement, and Gustavo Moura, Niteesh Bharara, and Siddharth Saraiya for help with developing the fitting software used in this work.

Supporting Information Available: Derivation of the cooperativity measure $\Xi$ and its relation to the Hill coefficient. Adair equation using microscopic constants. This material is available free of charge via the Internet at http://pubs.acs.org.

\section{References and Notes}

(1) Klotz, I. M. Ligand-Receptor Energetics; Wiley \& Sons Inc.: New York, 1997.

(2) Voet, D.; Voet, J. G. Biochemistry, 2nd ed.; J. Wiley and Sons: New York, 1995.

(3) Hammes, G. G.; Wu, C. W. Science 1971, 172, 1205-1211.

(4) Adair, G. S. J. Biol. Chem. 1925, 63, 529-538.

(5) Monod, J.; Wyman, J.; Changeux, J. P. J. Mol. Biol. 1965, 12, $88-118$.

(6) Koshland, D. E.; Nemethy, J.; Filmer, D. Biochemistry 1966, 5, $365-382$.

(7) Hill, T. L. Cooperativity theory in biochemistry; Springer-Verlag: New York, 1985.

(8) Wyman, J.; Gill, S. J. Binding and linkage; University Science Books: Mill Valley, CA, 1990.

(9) Klotz, I. M. Q. Rev. Biophys. 1985, 18, 227-259.

(10) Schellman, J. A. Biopolymers 1975, 14, 999-1018.
(11) Haiech, J.; Klee, G. B.; Demaille, J. G. Biochemistry 1981, 20, 3890-3897.

(12) Alberty, R. A. J. Phys. Chem. B 2000, 104, 9929-9934.

(13) Poland, D. J. Chem. Phys. 2000, 113, 4774-4784.

(14) Poland, D. Biopolymers 2003, 69, 60-71.

(15) Tanford, C. Adv. Prot. Chem. 1962, 17, 69-165.

(16) Miller, D. W.; Dill, K. A. Protein Sci. 1997, 6, 2166-2179.

(17) Alberty, R. A. Biophys. Chem. 1996, 62, 141-159.

(18) Owczarzy, R.; Dunietz, I.; Behlke, M. A.; Klotz, I. M.; Walder, J. A. Proc. Natl. Acad. Sci. U.S.A. 2003, 100, 14840-1485.

(19) Koper, G.; Borkovec, M. J. Phys. Chem. B 2001, 105, 66666674

(20) Wang, L. Y.; DiCera, E. Proc. Natl. Acad. Sci. U.S.A. 1996, 93, 12953-12958.

(21) Onufriev, A.; Case, D. A.; Ullmann, G. M. Biochemistry 2001, 40, 3413-3419.

(22) Ullmann, G. M. J. Phys. Chem. B 2003, 107, 1263-1271.

(23) Klotz, I. M.; Hunston, D. L. J. Biol. Chem. 1975, 250, 30013009 .

(24) Briggs, W. E. Biophys. Chem. 1983, 18, 67-71.

(25) Di Cerra, E. Thermodynamic theory of site-specific binding processes in biological macromolecules, 1st ed.; Cambridge University Press: New York, 1995; pp 169-190.

(26) Fletcher, J. E.; Spector, A. A.; Ashbrook, J. D. Biochemistry 1970, 9, 4580-4587.

(27) Perutz, M. F. Nature 1970, 228, 726-739.

(28) Chein, J. C. W.; Mayo, K. H. J. Biol. Chem. 1980, 225, 97909799.

(29) Connelly, P. R.; Robert, C. H.; Briggs, W. E.; Gill, S. J. Biophys. Chem. 1986, 24, 295-309.

(30) Briggs, W. E. J. Theor. Biol. 1985, 114, 605-614.

(31) Briggs, W. E. Rocky Mt. J. Math 1985, 15, 75-89.

(32) da Silva, J. F.; Williams, R. The Biological Chemistry of the Elements - The Inorganic Chemistry of Life; Oxford University Press: New York, 2001. 